\title{
3D Characterization of Silicon Based Electrode Material for Advanced Lithium-Ion Storage Technologies
}

\author{
T. Vorauer ${ }^{1}$, J. Rosc ${ }^{1}$, P. H. Jouneau ${ }^{2}$, P. Bayle-Guillemaud ${ }^{2}$, B. Fuchsbichler ${ }^{3}$, S. Koller ${ }^{3}$, R. Brunner ${ }^{1}$ \\ 1. Materials Center Leoben, Forschung GmbH, A8700 Leoben, Austria. \\ 2. Univ. Grenoble Alpes, CEA, DRF/INAC/MEM, F38054 Grenoble, France \\ 3. Varta Micro Innovation GmbH, A8010 Graz, Austria
}

Energy storage will be according to the European Energy Storage Technology Development Roadmap (EASE/EERA) towards 2030 [1], of high importance for worldwide climate energy objectives as it has the potential to cover the entire energy value chain. Especially lithium-based technology will play an important role in this context [1]. However, lithium (Li)-ion batteries still have to struggle with several shortcomings, mainly due to material deficiencies, leading to a limited lifetime and the still high energy costs per $\mathrm{kWh}$. In order to conquer these shortcomings, the use of novel material approaches and processing steps need to be investigated, which are also suitable for industrial applications. A breakthrough in this manner can be achieved e.g. by the use of, (1) carbon-based materials, (2) silicon( $\mathrm{Si}$ )-based material and (3) cost efficient electrode and cell manufacturing processes. However, still several scientific and technological questions for silicon based lithium-ion batteries e.g. the impact of various loading cycles, which reduce the life time of batteries and components and of storage capacity, are open. In this context especially the microstructure of the electrode material plays an important role since it has big impact on the performance on the battery. Therefore, experimental methods which include 2D and 3D from the electrode material over the $\mathrm{mm}$ to the $\mathrm{nm}$ scale as well as accurate image analyzing algorithm are necessary.

In this work we develop a characterization toolbox suitable to analyze the micro structure of pristine Si-based anode material which is relevant for future Li-ion batteries used in industrial related storage solutions. This comprises (1) the measurement of the microstructure by using focused ion beamscanning electron microscopy (FIB-SEM) and X-ray computed tomography (XCT) to cover the length scales from $\mathrm{mm}$ to $\mathrm{nm}$ including $2 \mathrm{D}$ and $3 \mathrm{D}$ information, (2) the development of suitable image analysis algorithm to obtain accurate results regarding the microstructure of the electrode, and (3) the validation of the data by comparing FIB-SEM and XCT results.

The active region of the sample is basically defined by a graphite matrix, a binder and the distributed $\mathrm{Si}$ particles within the graphite/binder matrix. The electrode material is manufactured by mixing the components directly and cast them for instance on a copper foil. Samples are then prepared for the individual methods. In addition we compare different samples having different weight percentage of the Si phase. The XCT system has a focal spot size of $800 \mathrm{~nm}$ and gives the possibility to measure for these particular samples a Voxel size $V$ down to about $V_{x}=V_{y}=V_{z}=700 \mathrm{~nm}$. This gives the opportunity to gain 3D information between the $\mathrm{mm}$ to the $\mu \mathrm{m}$ scale. For the $\mu \mathrm{m}$ to the $\mathrm{nm}$ scale we use FIB-SEM. For the analysis of the 2D and 3D structural data we discuss the use of the commercially available imaging software Avizo ${ }^{\circledast}$ from FEI and Python. For the segmentation of the different phases of the electrode material we are using an approach to iterate all possible threshold intervals and apply segmentation algorithms including watershed and random walker [2] to each interval. This should provide the possibility that the properties of the unique components should not change within a threshold interval i.e. each class of particles corresponds to a threshold interval. In order to provide guidelines for the 
optimum electrode configuration we analyze, the geometric properties of the electrode, e.g. the thickness, the porosity, graphite and binder content, the Si particle volume and size distribution, the maximum and minimum distance between the center of mass of the particles (Fig.1). For the validation of the methods the different results are compared. The findings shall give information of how the Siparticles are distributed within the active material for a certain production process. Further studies to relate the findings of the microanalysis to the storage capacity are planned for the future.

\section{References:}

[1] European Energy Storage Technology Development Roadmap Towards 2030 EERA (2013). http://www.eera-set.eu/wp-content/uploads/148885-EASE-recommendations-Roadmap-04.pdf

[2] Serge Beucher and Christian Lantuéjoul workshop on image processing, real-time edge and motion detection (1979). http://cmm.ensmp.fr/ beucher/publi/watershed.pdf; Leo Grady, Random Walks for Image Segmentation, IEEE Transactions on Pattern analysis and machine intelligence, vol. 28, no. 11, Nov. 2006

[3] This work has received funding from the European Union (EU) under the Horizon 2020 research and innovation program (grant agreement no. 685716 "SINTBAT"). Financial support by the Austrian Federal Government (in particular from Bundesministerium für Verkehr, Innovation und Technologie and Bundesministerium für Wissenschaft, Forschung und Wirtschaft) represented by Österreichische Forschungsförderungsgesellschaft $\mathrm{mbH}$ and the Styrian and the Tyrolean Provincial Government, represented by Steirische Wirtschaftsförderungsgesellschaft $\mathrm{mbH}$ and Standortagentur Tirol, within the framework of the COMET Funding Programme (No. 837900) is also gratefully acknowledged.

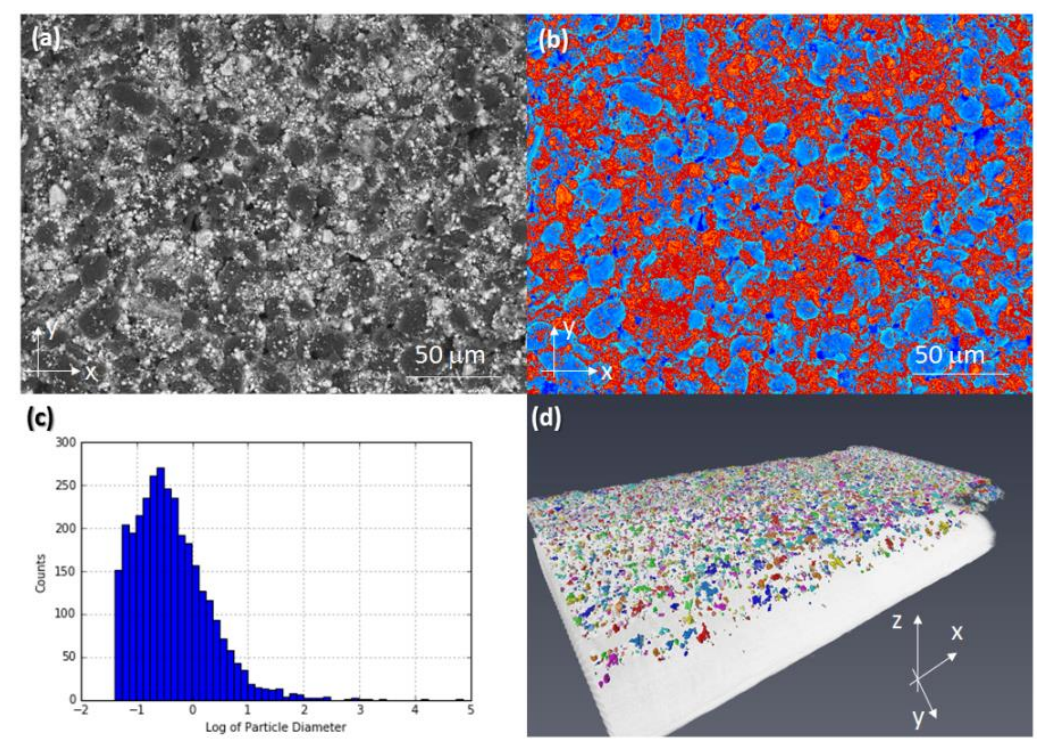

Figure 1. (a) BSE image obtained from electrode material. The different grey values correspond to the different phases, mainly graphite, pores, binder and Si particles. (b) Microanalysis of the BSE image using the developed segmentation algorithm. Here, the particles are segmented and highlighted in red. (c) Histogram showing the size distribution from the Si-particles in the active material obtained from the image analysis. (d) 3D XCT image at $\mathrm{V}_{\mathrm{x}}=\mathrm{V}_{\mathrm{y}}=\mathrm{V}_{\mathrm{z}}=700 \mathrm{~nm}$ showing the segmented Si particles within the graphite-binder compound. 